\title{
The Opioid Epidemic: Impact on Children and Families
}

\section{April Dirks*}

Social Work Program, Mount Mercy University, Cedar Rapids, IA 52402, United States

*Corresponding Author: Dr. April Dirks, Social Work Program, Mount Mercy University, Cedar Rapids, IA 52402, United States, Tel: 319-363-8213 Ext 1385; E-mail: adirks@ mtmercy.edu

Received: 16 December 2017; Accepted: 09 January 2018; Published: 15 January 2018

\section{The Opioid Epidemic: Impact on Children and Families}

A national focus on opioid abuse has heightened public awareness of the extent and severity of drug addiction and brings to question the impact opioid use has on children and families. The term opioid can be used when referring to a wide array of prescription pain medications and illegal substances such as heroin. While there are several types of opioids being abused, the focus of this review will be primarily on the abuse of opiate pain medications due to the alarming rate of overdose that has resulted in the deaths of 44 people each day in the United States (Centers for Disease Control and Prevention [CDC] [1]. Addiction to prescription pain medications has had a devastating effect on families as exemplified by the increasing number of infants born to drug addicted mothers and children who reside in homes with parents addicted to opioid pain medications. This review will examine the opioid epidemic and the effect of parent drug use on children and families.

According to the National Institute on Drug Abuse regular use of opioid pain relievers are generally safe when taken for a short time and prescribed by a doctor but, because they produce a sense of euphoria in some patients, they can easily be misused or abused causing drug dependency [2]. Regular use, even when used as prescribed by a doctor, can lead to dependence, overuse, and even death. Opioid drug dependence is a national health concern because Americans consume $80 \%$ of the world's supply of opioid pain medications [3]. Even more alarming, Americans consume $99 \%$ of the prescription drug Hydrocodone produced worldwide, which is a synthetic opioid derived from the pharmaceutical drug codeine and is very potent and addictive. The opioid crisis in the United States is believed to have stemmed from the increased awareness of chronic pain by physicians. This, coupled with the push from pharmaceutical companies for providers to prescribe opioid medications, led to a drastic increase of opioid use among Americans in the last two decades. Many Americans believe that a medication that is prescribed by their physician must be safe for use to manage pain. This belief, combined with the euphoric feeling provided by the drugs, can lead to misuse of the medication and long-term dependence. 
The misuse of opioid pain medications can affect not only the user, but can harm those around them. For example, a pregnant mother may continue opioid drug use during pregnancy and this can have a negative effect on the unborn child. Women who are addicted to opioid pain killers may choose to forego necessary prenatal care for fear that the healthcare provider may learn of their addiction. Many opioid users also suffer from nutritional neglect, which could have a negative impact on the fetus if a woman is pregnant. The number of pregnant women using narcotic pain medications rose five-fold from 2000-2009 and continues to rise [3]. The increase has resulted in infants being born with a condition called Neonatal Abstinence Syndrome (NAS) that is linked to birth defects and severe withdrawal symptoms demonstrated by the infant at birth.

Children born with NAS may experience a variety of symptoms ranging from nausea, vomiting, tremors or seizures [4]. These symptoms, along with other early signs such as a restless infant with high pitched cries, may appear immediately after birth or take several days to develop. Infants with NAS may be difficult to feed and the withdrawal symptoms they experience can ensue for as long as four to six months. Over half of the women who abuse opioids during pregnancy will give birth to a child with these symptoms. In America, the number of infants born addicted to opioids is growing and it is thought that 6 out of every 1,000 births are linked to NAS [5].

Treatment for symptoms of NAS range from non-medical interventions such as swaddling the infant and limiting environmental stimuli to the use of medications such as methadone or morphine to ween infants off of the opioids in their system. While the immediate side-effects of the syndrome, such as seizures, are concerning it is also thought that children with severe opioid addiction could have long-term developmental delays and permanent learning disabilities and other difficulties [4]. An infant may suffer from disordered brain development while in utero while the mother was using and may also have difficulty bonding with others and develop attachment disorders as a result of their mothers drug use. Because of their special needs, many children with NAS will end up in the foster care system. In fact, the number of children in foster care as a result of the opioid epidemic has risen 8 percent from 2012 to 2015 [5]. With the increased rates of children being born to addicted mothers, it is expected that the number of children in foster care will continue to grow, increasing the strain on the already limited foster care system.

Parents addicted to opioids may suffer from a Substance Use Disorder (SUD) and it is estimated that approximately 8 million children under the age of 18 live with at least one adult who has this disorder, with the majority of these children being under the age of five [6]. The home environment of these families is often unstable and children are exposed to a variety of experiences that result in secrecy, loss, conflict, violence, and fear. Children who live with a parent who has a SUD may live in a chaotic environment and have little or no stability due to their parent's drug addiction. They are impacted by their parents drug use on many levels and may experience emotional, behavioral, or substance abuse problems themselves. Substance abuse can occur at any stage of life but one risk factor that increases the likelihood an adult will abuse drugs includes a previous family history of living with a drug user [3]. Therefore, it can be said that living with an opioid addicted parent will have a negative impact on children living in the home and increase the likelihood they could develop a SUD themselves. 
Parents with SUD are three times more likely to physically or sexually abuse their children, resulting in additional strain to the already traumatized child living in a home with a substance abuser [6]. In addition to risk factors for child abuse, children living with an addicted parent are often subjected to a caregiver whose mood may be altered, they may be preoccupied with drug use, or may need significant amounts of time to recover from drug use. This may result in a child with missed opportunities to foster a healthy attachment with their parent. Older children may experience a role reversal as they take on the role of caregiver for the younger children, filling the void of a parent who is emotionally or physically absent. Without healthy attachment to a caregiver, a child is much more vulnerable to trauma and stress and may have problems with depression, anxiety or other mental health disorders [7].

It has been demonstrated that opioid addiction can have negative effects not only on the user, but on the entire family system. Infants born to a drug addicted mother may suffer long-term health effects and developmental delays. Older children who live with a parent addicted to opioid pain medications may also suffer neglect or child abuse in the care of a parent abusing pain medications. The opioid epidemic has reached a crisis level drawing the attention of national media and social service agencies trying to reduce a trend of pharmaceutical drug abuse. It is essential that the health and well-being of children also be considered when discussing the opioid epidemic in order to increase the quality of life for all family members living with an addicted adult.

\section{References}

1. Centers for Disease Control and Prevention. Opioid Overdose (2017).

2. National Institute on Drug Abuse. Opioids (2017).

3. Blozen B. Prescription opioids: A growing epidemic of abuse. Journal of American Nurses 8 (2013): 38 -43.

4. Thompson D. Health Day Reporter: Opioid addicted babies may struggle with learning (2017).

5. Rappleye H, McHugh R, Farrow R. Born addicted: The number of opioid addicted babies is soaring (2017).

6. Lander L, Howsare J, Byrne M. The impact of substance use disorders on families and children: From theory to practice. Social Work in Public Health 28 (2013): 194-205.

7. Slesnick N, Feng X, Brakenhoff B, et al. Parenting under the influence: The effect of opioids, alcohol and cocaine on mother-child interaction. Addictive Behaviors 39 (2014): 897-900. 